\title{
EDAD DE CASTRACIÓN Y SU EFECTO SOBRE EL DESEMPENOO PRODUCTIVO DE CERDOS CRUZADOS EN FASE DE CEBA
}

\author{
CASTRATION AGE AND ITS EFFECT ON \\ PRODUCTIVE PERFORMANCE OF CROSSBRED \\ PIGS IN THE FATTENING PHASE
}

\author{
Libardo Maza A. ${ }^{1 *}$, Juan Simanca Sotelo ${ }^{2}$, Orlando Narváez Diaz ${ }^{3}$, Carlos Almentero Suárez ${ }^{4}$, Óscar Vergara G. ${ }^{5}$
}

\begin{abstract}
${ }^{1}$ MVZ, M.Sc. Universidad de Córdoba, Facultad de Medicina Veterinaria y Zootecnia, Grupo de Investigación en Producción Animal Tropical. Montería, Colombia. *autor para correspondencia. E-mail: Imaza@correo.unicordoba.edu.co; ${ }^{2}$ MVZ, M.Sc. Instituto Nacional de Vigilancia de Medicamentos y Alimentos - INVIMA, Grupo de Investigación en Producción Animal Tropical, Montería, Colombia; e-mail: lj.c.simanca@hotmail.com; ${ }^{3}$ MVZ. Ejercicio particular, Grupo de Investigación en Producción Animal Tropical. Montería, Colombia, e-mail: orlandomvz@hotmail.com; ${ }^{4}$ MVZ, Esp. Universidad de Córdoba, Facultad de Medicina Veterinaria y Zootecnia, Grupo de Investigación en Producción Animal Tropical. Montería, Colombia, e-mail: calmentero@correo.unicodoba.edu.co; ${ }^{5}$ Zoot, Dr. Sc. Universidad de Córdoba, Facultad de Medicina Veterinaria y Zootecnia, Grupo de Investigación en Producción Animal Tropical. Montería, Colombia, e-mail: overgara@correo.unicordoba.edu.co
\end{abstract}

Rev. U.D.C.A Act. \& Div. Cient. 20(1): 215-219, Enero Junio, 2017

\section{INTRODUCCIÓN}

La castración quirúrgica en cerdos, a temprana edad, es la técnica más utilizada, mundialmente, para eliminar el olor sexual que se presenta en la pubertad, durante la etapa de engorde, además de controlar el comportamiento agresivo de los machos enteros (Fábrega et al. 2009, Bahelka et al. 2015). Además de ello, la producción de cerdos para sacrificio se basa, fundamentalmente, en machos castrados y hembras, aunque trae, como inconveniente, un mayor acumulo de grasa corporal (Peinado et al. 2008; Meier-Dinkel et al. 2015)

La edad de la castración puede afectar al ritmo de crecimiento de los animales. El mayor crecimiento del tejido muscular de los machos enteros es más evidente, a medida que se aproximan a la pubertad (Quiles, 2009). Considerando que en los sistemas de producción porcina del trópico bajo colombiano no existe un criterio definido para la edad de castración de los lechones, el presente estudio tuvo como objetivo evaluar el efecto de la edad de castración sobre la ganancia diaria de peso, la conversión alimenticia y el rendimiento de canal caliente de cerdos mestizos, en fase de ceba.

\section{MATERIALES Y MÉTODOS}

Sitio de estudio y geoclima. El estudio, se realizó en la Granja experimental de la Facultad de Medicina Veterinaria y Zootecnia, de la Universidad de Córdoba, localizada en el corregimiento de Berastegui, Municipio de Ciénaga de Oro, departamento de Córdoba, Colombia. La zona, se encuentra clasificada como bosque húmedo tropical, ubicado a $8^{\circ}$ 52' latitud Norte y $75^{\circ} 54^{\prime}$ de longitud al Oeste, altura a $18 \mathrm{msnm}$, temperatura promedio de $27^{\circ} \mathrm{C}$, humedad relativa de $85 \%$ y precipitación anual de $1.200 \mathrm{~mm}$.

Unidades experimentales y tratamientos. Se utilizaron 16 cerdos machos cruzados (25\% Pietrain, 25\% Duroc, 25\% Landrace y 25\% Large White), con peso promedio inicial de $21,44 \pm 0,97 \mathrm{~kg}$ y con 58 a 60 días de edad. Los animales fueron distribuidos al azar, en cuatro grupos de tratamientos de cuatro animales cada uno, a saber: T0 = castrados al día 30 de edad; $\mathrm{T} 1$ = castrados al día 60 de edad; $\mathrm{T} 2$ = castrados al día 90 de edad y T3 = castrados al día 120 de edad.

Los animales fueron castrados quirúrgicamente y, para ello, cada animal fue sedado mediante inyección intramuscular profunda de $2 \mathrm{mg}$ de Azaperona ${ }^{\circledR}$ por $\mathrm{kg}$ de peso vivo. La técnica quirúrgica incluyó: lavado y desinfección con yodóforo de la zona testicular y periférica; inyección de, aproximadamente, $10 \mathrm{~mL}$ de anestésico local (lidocaína) en el área a cortar; corte con bisturí de la piel del escroto y las túnicas vaginal, parietal y visceral; amarre del cordón espermático con hilo de sutura desinfectado; corte del cordón espermático y extracción del testículo; extracción completa de túnicas testiculares; sutura del corte escrotal, dejando un espacio en la parte inferior para drenaje de líquidos y, por último, lavado y nueva desinfección del área intervenida. Al final de la 
cirugía, los machos recibieron una inyección de antibiótico (penicilina $15.000 \mathrm{Ul} / \mathrm{kg}$ de peso) y de antiinflamatorio (betametazona $1 \mathrm{~mL} / 20 \mathrm{~kg}$ de peso).

Manejo de los animales y la nutrición. Los animales fueron manejados en corrales individuales, para poder determinar el consumo de alimento por tratamiento. Los cerdos estuvieron sometidos a un periodo de adaptación al manejo experimental de 10 días, antes de entrar al experimento. La dieta cumplió con todos los requerimientos nutricionales de cada etapa productiva de la fase de ceba (levante, desarrollo y acabado), según las tablas de requerimientos nutricionales, propuestas por NRC (2001).

La cantidad de alimento suministrado, de acuerdo al ajuste que se hacía por peso de los animales, fue de $2,06 \mathrm{~kg} / \mathrm{animal} /$ día, para la fase de levante; $2,66 \mathrm{~kg} / \mathrm{animal} / \mathrm{día}$, para desarrollo y $3,3 \mathrm{~kg} /$ animal/día, para acabado. Con esta cantidad, se aseguró un consumo total de 1,$79 ; 2,31$ y $2,87 \mathrm{~kg} /$ animal/ día, para la fase de levante, de desarrollo y de acabado, respectivamente, de materia seca. El alimento fue suministrado en tres porciones a horas exactas (8:00 a.m., 12:00 m. y 4:00 p.m.).

El sacrificio de los animales, se realizó el día 150 de edad, en la sección de cárnicos de la Planta Piloto del Programa de Ingeniería de Alimentos, de la Universidad de Córdoba.

Ganancia diaria de peso (GDP). Para determinar la GDP de los animales, se realizaron pesajes semanales, a las 6:00 a.m., antes de iniciar el suministro de alimentos. Para el pesaje, se empleó una báscula de $300 \mathrm{~kg}$ de capacidad y $100 \mathrm{~g}$ de intervalo de precisión.

Conversión alimenticia (CA). La CA, se determinó dividiendo el consumo de alimento diario entre la ganancia de peso diaria promedia, determinada para cada tratamiento $(\mathrm{CA}=\mathrm{Consumo/GP)}$. Este parámetro, se estimó cada semana, en la que se realizaba el pesaje.

Rendimiento de canal caliente (RCC). Para evaluar el RCC, después del sacrificio de los animales, las canales se pesaron en una báscula digital de $200 \mathrm{~kg}$ de capacidad. El RCC, se estimó con base en la siguiente fórmula: (PCC/PV*100), donde, PCC correspondió al peso de la canal caliente y PV, al peso vivo del animal.

Análisis estadístico. Los resultados correspondientes a la GDP, CA y RCC fueron analizados, a través de un análisis de varianza y para la comparación de medias, se utilizó la prueba de Duncan. La variable RCC fue transformada mediante la expresión arcsen de la raíz cuadrada del porcentaje, para que cumpliera el supuesto de normalidad. Además, se utilizó la prueba de polinomios ortogonales, para determinar la tendencia de las variables respuestas, según la edad de castración. Los datos fueron analizados usando el programa SAS (2001).

Aspectos éticos. Los procedimientos quirúrgicos realizados en este estudio fueron ejecutados por el docente del área de Cirugía de la Facultad de Medicina Veterinaria y Zootecnia, de la Universidad de Córdoba y se tuvo en cuenta las normas éticas, técnicas, científicas y administrativas, para la investigación en animales, según lo mencionado en la ley 84 (Congreso de la República de Colombia, 1989) y lo referido por MRAD (2006).

\section{RESULTADOS Y DISCUSIÓN}

Ganancia diaria de peso (GDP). Se encontró diferencia altamente significativa $(P \leq 0,05)$ para la GDP, entre los diferentes tratamientos. T0 y T1 fueron estadísticamente iguales $(P>0,05)$ y difirieron $(P \leq 0,05)$ de T2 y T3 (Tabla 1$)$. Al hacer la prueba de polinomios ortogonales, se encontró que la GDP tuvo un comportamiento lineal $(P \leq 0,01)$ respecto a las edades de castración, según la siguiente ecuación: GDP $=640,6-0,66$ días $\left(R^{2}=0,52\right)$. Esto sugiere que, a medida que aumenta la edad de castración, disminuye la ganancia diaria de peso. Como se puede observar en la gráfica 1(A), hubo una disminución en la ganancia de peso al final del periodo experimental en T2 y T3, que pudo ser causado por el estrés posquirúrgico, al castrar a los animales de estos tratamientos, a los 90 y 120 días de edad.

Las GDP del presente estudio fue inferior a la reportada por Peinado et al. (2008), en cerdos Pietrain - Large White $x$ Landrace - Large White, castrados a los 7 días (746g/día); a la reportada por Bahelka et al. (2015), en cerdos Yorshire $\mathrm{x}$ Landrace, castrados a los 7 días de edad $(890 \pm 606 \mathrm{~g} /$ día); a la hallada por Daza et al. (2016), en cerdos Duroc x Landrace x Large White, castrados a los 5 días de edad (993g/día) y a la estimada por Van den Broeke et al. (2016), en animales Pietrain cruzados, castrados a los 5 días (877 g/día). De igual manera, Escobar et al. (2006), en cerdos PIC x LandraceYorkshire-Duroc, reportaron GDP entre 705 a 848 g/día, en cerdos castrados a los 30 días de edad, nutridos con alimento balanceado comercial y diferentes niveles de melaza.

Conversión alimenticia (CA). Se encontró que la CA fue menor en T0 y T1 $(3,87$ y 3,93) y mayor en T2 y T3 $(4,39$ y 4,31) $(\mathrm{P} \leq 0.05)$ (Tabla 1). Esta diferencia puede ser atribuida al mayor estrés provocado por la mayor edad a la castración del presente estudio, traduciéndose en una disminución en el desempeño productivo del animal (Lanier, 2008). Al realizar la prueba de polinomios ortogonales, esta variable no tuvo ningún tipo de comportamiento polinomial. En la gráfica 1(B), se puede ver el comportamiento de la conversión alimenticia, durante el periodo experimental. 
Tabla 1. Comportamiento de la ganancia diaria de peso $(\mathrm{kg})$, conversión alimenticia $(\%)$ y rendimiento promedio en canal caliente (\%) de cerdos, con diferentes edades a la castración.

\begin{tabular}{|c|c|c|c|}
\hline Tratamientos & Ganancia Diaria de Peso (g) & $\begin{array}{c}\text { Conversión } \\
\text { Alimenticia }\end{array}$ & $\begin{array}{c}\text { Rendimiento en Canal } \\
\text { Caliente \% }\end{array}$ \\
\hline T0 & $622^{\mathrm{a}}$ & $3,87^{\mathrm{a}}$ & $71,79^{\mathrm{a}}$ \\
\hline T1 & $606^{\mathrm{a}}$ & $3,93^{\mathrm{a}}$ & $71,19^{\mathrm{ab}}$ \\
\hline T2 & $564^{\mathrm{b}}$ & $4,39^{\mathrm{b}}$ & $68,92^{\mathrm{b}}$ \\
\hline T3 & $570^{\mathrm{b}}$ & $4,31^{\mathrm{b}}$ & $68,58^{\mathrm{b}}$ \\
\hline
\end{tabular}

Valores medios con diferente letra en la misma columna, difieren estadísticamente $(P \leq 0,05)$.

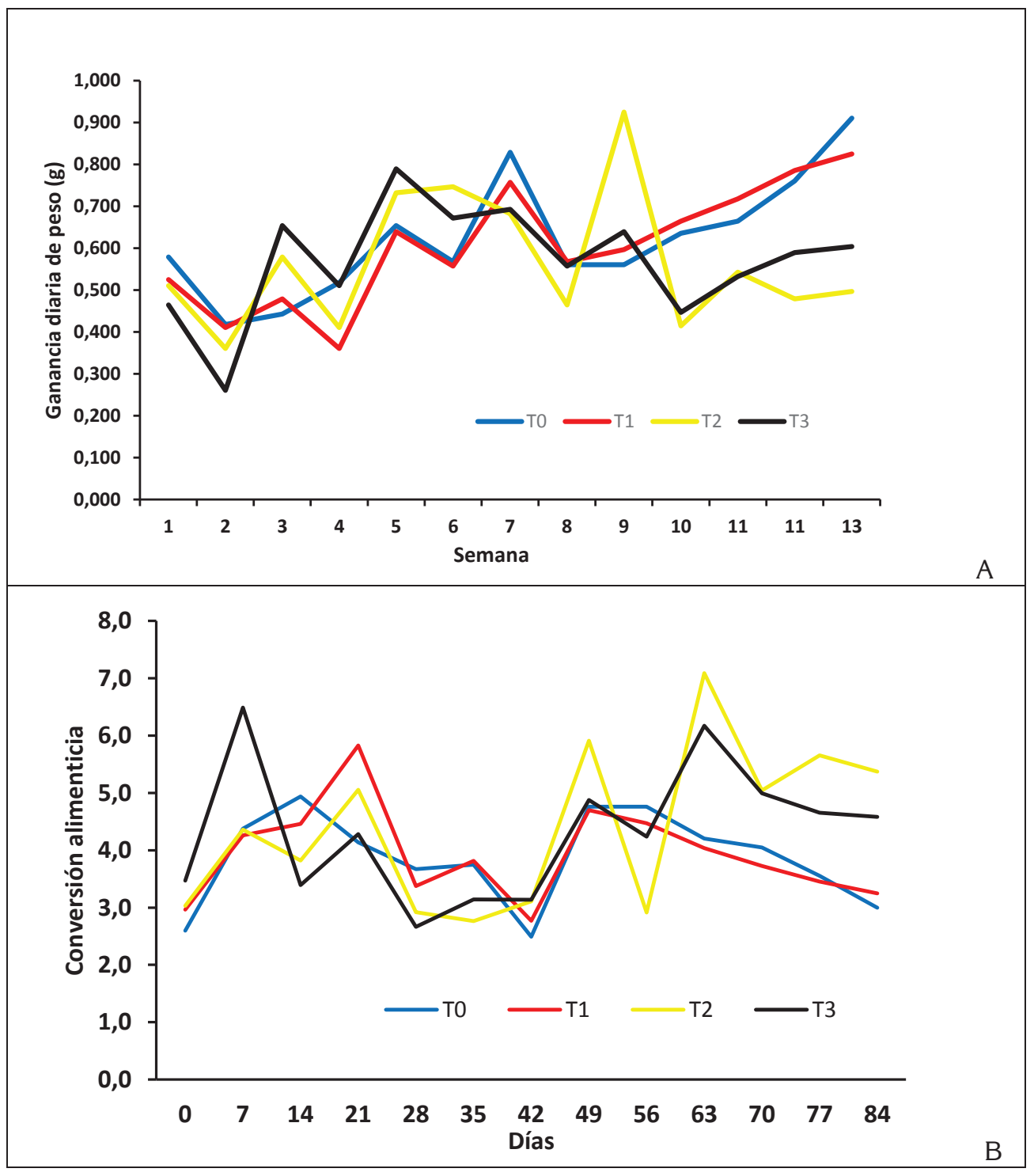

Gráfica 1. A) Comportamiento de la ganancia de peso y B) conversión alimenticia, para los diferentes tratamientos durante el periodo experimental. 
La CA del presente estudio fue menor $(3,87$ - 4,31) a la reportada $(4,52)$ por Gómez et al. (2013), en cerdos Ibéricos, castrados a los 60 días de edad, mientras que fue superior a la reportada por Fábrega et al. (2009), Morales et al. (2010), Bahelka et al. (2015) y Daza et al. (2016), en cerdos castrados a diferentes edades $(2,77,2,56,2,86$ y 2,99, respectivamente). Esta mayor CA en los resultados del presente estudio, se puede atribuir a las condiciones ambientales del trópico bajo donde fue realizado el experimento, lo cual, afecta, de manera directa, el consumo de alimento, la ganancia de peso y, por ende, la conversión; además, de las líneas genéticas y alimento utilizado.

Rendimiento de canal caliente (RCC). Los promedios de RCC de los diferentes tratamientos oscilaron entre 68,58 a $71,79 \%$. Se encontró que T0 obtuvo el mayor rendimiento en canal caliente y difirió $(\mathrm{P} \leq 0,05)$ de T2 y T3 (Tabla 1). Estos resultados están de acuerdo a los reportados por Escobar et al. (2006), quienes suministraron diferentes niveles de melaza (0, 10, 20 y 30\%) al alimento balanceado comercial, en cerdos en fase de ceba y castrados a los 30 días de edad, encontrado RCC de 66,6; 69,1; 70,6 y 67,5\%, respectivamente, para los diferentes niveles de melaza. Valores superiores al presente estudio han sido reportados por Fonti et al. (2012), en cerdos (Duroc) y Van den Broeke et al. (2016), en cerdos Pietrain cruzados con RCC, de $76,9 \pm 0,25$ y $79 \pm 0,3 \%$, respectivamente.

Al realizar la prueba de polinomios ortogonales, se encontró que el $R C C$ tuvo un comportamiento lineal $(P \leq 0,01)$ respecto a las edades de castración, según la siguiente ecuación: $\mathrm{RCC}=73,09-0,04$ días $\left(\mathrm{R}^{2}=0,44\right)$. Esto sugiere que, a medida que aumenta la edad de castración, disminuye el rendimiento en canal caliente de los animales.

La GDP fue mayor y la CA fue menor en los animales castrados a 30 días de edad (T0) y 60 días de edad (T1); sin embargo, se puede presumir que el mejor tratamiento fue el testigo (T0), ya que pudo presentar menor estrés posquirúrgico. El RCC fue similar entre los tratamientos y, por ende, su comportamiento es independiente a la edad a la castración.

Agradecimientos. Los autores agradecen a la Facultad de Medicina Veterinaria, de la Universidad de Córdoba, por facilitar los animales para la realización de este estudio. Además, a los MVZ Jorge Guzmán y Sonnel Velásquez. Conflicto de intereses: El manuscrito fue preparado y revisado con la participación de todos los autores quienes declaramos que no existe conflicto de intereses que ponga en riesgo la validez de los resultados presentados.

\section{BIBLIOGRAFÍA}

1. BAHELKA, I.; TOMKA, J.; BUČKO, O.; HANUSOVÁ, E. 2015. Growth performance and carcass quality of entire males, surgical castrates and gilts. Slovak J. Anim. Sci. 48(3):116-121.

2. CONGRESO DE LA REPÚBLICA DE COLOMBIA. 1989. Ley 84 de 1989. Por la cual se adopta el Estatuto Nacional de Protección de los Animales y se crean unas contravenciones y se regula lo referente a su procedimiento y competencia. Disponible desde Internet en: http:/www.alcaldiabogota.gov.co/sisjur/normas/ Norma1.jsp?i=8242. (con acceso 08/04/2017).

3. DAZA, A.; LATORRE, M.A.; OLIVARES, A.; LÓPEZ C.J. 2016. The effects of male and female immunocastration on growth performances and carcass and meat quality of pigs intended for dry-cured ham production: A preliminary study. Livest. Sci. 190: 20-26.

4. ESCOBAR, J.; MACÍAS, M.; CASTILLO, R.; VÉLEZ, M. 2006. Evaluación del uso de melaza en dietas para cerdos en crecimiento y engorde. Ceiba. 47(1-2):39.

5. FÁBREGA, E.; SOLER, J.; CROS, J.; GRISPERT, M.; TIBAU, J.; VELARDE, A. 2009. Resultados de diversas alternativas a la castración quirúrgica en cerdos. SUIS. 59:26-34.

6. FONTI-FURNOLS, M.; GISPERT, M.; SOLER, J.; DIAZ, M.; GARCIA-REGUEIRO, J.A.; DÍAZ, I.; PEARCE, M.C. 2012. Effect of vaccination against gonadotrophin-releasing factor on growth performance, carcass, meat and fat quality of male Duroc pigs for dry-cured ham production. Meat Sci. 91:148-154.

7. GÓMEZ-FERNÁNDEZ, J.; HORCAJADA, S.; TOMAS, C.; GÓMEZ-IZQUIERDO, F.; DE MERCADO, E. 2013. Efecto de la inmunocastración y de la castración quirúrgica sobre los rendimientos productivos y de la calidad de la canal en cerdas ibéricas de cebo. ITEA. 109(1):33-48.

8. LANIER, J. 2008. El estrés y el miedo en procedimientos estándares agropecuarios. REDVET. 9(10B):1-13. Disponible desde Internet en: http://www.veterinaria. org/revistas/redvet/n101008B/BA029.pdf (con acceso $08 / 03 / 2017)$.

9. MEIER-DINKEL, L.; GERTHEISS, J.; MULLER, S.; WESOLY, R.; MORLEIN, D. 2015. Evaluating the perfor- 
mance of sensory quality control: the case of boar taint. Meat Sci. 100:73-84.

10. MRAD, A. 2006. Ética en la investigación con modelos animales experimentales. Alternativas y las 3 RS de Russel. Una responsabilidad y un compromiso ético que nos compete a todos. Rev. Col. Bioét. 1(1):163184.

11. MORALES, J.; GISPERT, M.; HORTOS, M.; PÉREZ, J.; SUÁREZ, P.; PIÑEIRO, C. 2010. Evaluation of production performance and carcass quality characteristics of boars immunised against gonadotropin releasing hormone $(\mathrm{GnRH})$ compared with physically castrated male, entire male and female pigs. Span. J. Agric. Res. 8(3):599-606.

12. NATIONAL RESEARCH COUNCIL-NRC-. 2001. "10 nutrient requirement tables." Nutrient Requirements of Swine: 10th Revised Edition. Washington, DC: The National Academies Press. Disponible desde Internet en: http://www.nap.edu/openbook.php?record $\mathrm{id}=6016 \&$ page $=111$ (con acceso 10/02/2014).
13. PEINADO, J.; MEDEL, P.; FUENTETAJA, A.; MATEOS, G. 2008. Influence of sex and castration of females on growth performance and carcass and meat quality of heavy pigs destined for the dry-cured industry. J. Anim. Sci. 86:1410-1417.

14. QUILES, A. 2009. Castración de lechones: Ventajas e inconvenientes. Cría y salud porcina. 24:54-63.

15. STATISTICAL ANALYSIS SYSTEMS -SAS-. 2001. User's guide (version 9.1) Cary (USA). Institute SAS/STAT.

16. VAN DEN BROEKE, A.; LEEN, F.; ALUWÉ, M.; AMPE, B.; VAN MEENSEL, J.; MILLET, S. 2016. The effect of GnRH vaccination on performance, carcass, and meat quality and hormonal regulation in boars, barrows, and gilts. J. Anim. Sci. 94:2811-2820.

Recibido: Junio 3 de 2014

Aceptado: Mayo 30 de 2017

Cómo citar:

Libardo Maza A.; Simanca Sotelo, J.; Narváez Díaz, O.; Almentero Suárez, C.; Vergara G., Ó. 2017. Edad de castración y su efecto sobre el desempeño productivo de cerdos cruzados en fase de ceba. Rev. U.D.C.A Act. \& Div. Cient. 20(1): 215-219. 